\title{
Dipole-Dipole Interactions in Spin-Labeled Au Nanoparticles as a Measure of Interspin Distances
}

Petre Ionita, Agneta Caragheorgheopol, Bruce C. Gilbert and Victor Chechik

\section{Supplementary information.}

\section{Average distance between nearest neighbors on a sphere.}

The function $w(x)$ is defined so that $w(x) d x$ is the probability that for a given spin label, the distance to the nearest neighbor is between $x$ and $x+d x$. Equation (1s) follows from the main text of the paper.

$w(x) d x=($ probability of finding a label in zone 2$) \times($ probability that all other labels are in zone 3$)$

The probability that there are two labels in infinitesimally small zone 2 is infinitesimally small compared to the probability that there is one spin label in zone 2; the former can thus be ignored. One spin label is in position A (Figure 1s); there are $y$-1 spin labels remaining. The probability of finding a spin label in zone 2 is thus defined by the binomial distribution function ${ }^{y-1} C_{1} \cdot p(1-p)^{y-2}=(y-1) p(1-p)^{y-2}$, where $p$ is the ratio of the area of zone 2 to the area of the overall nanoparticle. As the ratio of the areas is infinitesimally small, this equation can be simplified as $(y-1) p=(y-1) \frac{\text { area of zone } 2}{\text { area of nanoparticle }}$. Similarly, the probability that all remaining $(y-2)$ spin labels are in zone 3 equals $p^{y-2}$.Equation (1s) can thus be re-written as (2s).

$$
w(x) d x=(y-1) \frac{\text { area of zone } 2}{\text { area of nanoparticle }} \times\left(\frac{\text { area of zone } 3}{\text { area of nanoparticle }}\right)^{y-2}
$$

The area of nanoparticle is $4 \pi r^{2}$, where $r$ is the nanoparticle radius. The area of zone 2 equals $2 \pi r\left(h_{2}-h_{1}\right)$ (Figure 1s).
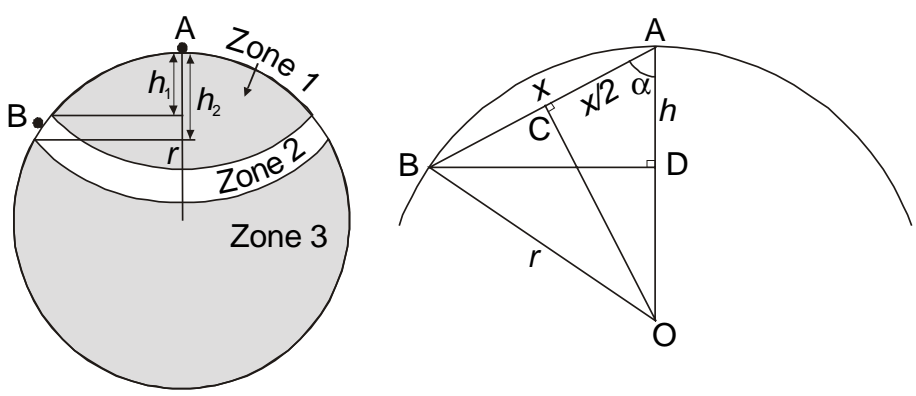

Figure 1s. Schematic drawing of a spin-labeled nanoparticle.

Let us now consider triangles $\mathrm{ABD}$ and $\mathrm{AOC}$. They are both right-angled, and they share the same angle $\alpha$. Hence, these triangles are similar, and $\mathrm{AD} / \mathrm{AB}=\mathrm{AC} / \mathrm{AO}$ or $h / x=\mathrm{AC} / r$. As the triangle $\mathrm{AOB}$ is isosceles, the altitude $\mathrm{OC}$ is also a median; hence $\mathrm{AC}=x / 2$. Substituting this relationship into the equation for similar triangles, we obtain $h / x=x / 2 r$, or $h=x^{2} / 2 r$. The area of the infinitesimally small zone 2 is therefore $2 \pi r\left(h_{2}-h_{1}\right)=\pi \cdot\left(x_{2}{ }^{2}-x_{1}{ }^{2}\right)=\pi \cdot\left(x_{2}-x_{1}\right) \cdot\left(x_{2}+x_{1}\right)=\pi \cdot d x \cdot 2 x=2 \pi x d x$. Similarly, the area of zone 3 could be written as area of the whole nanoparticle minus area of zone 1: $4 \pi r^{2}-2 \pi r h=4 \pi r^{2}-$ $2 \pi r \cdot x^{2} /(2 r)=\pi\left(4 r^{2}-x^{2}\right)$. Substituting these equations into equation (2s), we obtain relationship (3s) which after a simple transformation can be written as (4s). 


$$
\begin{aligned}
& w(x) d x=(y-1) \frac{2 \pi x d x}{4 \pi r^{2}} \times\left(\frac{\pi\left(4 r^{2}-x^{2}\right)}{4 \pi r^{2}}\right)^{y-2} \\
& w(x)=\frac{(y-1)}{2^{2 y-3} r^{2 y-2}} x\left(4 r^{2}-x^{2}\right)^{y-2}
\end{aligned}
$$

The average distance between nearest neighbors is given by equation $(5 \mathrm{~s})$.

$$
\bar{x}=\int_{0}^{2 r} x w(x) d x=\frac{(y-1)}{2^{2 y-3} r^{2 y-2}} \int_{0}^{2 r} x^{2}\left(4 r^{2}-x^{2}\right)^{y-2} d x
$$

The definite integral in (5s) can be expressed via the Gamma function to give (5s).

$$
\int_{0}^{2 r}\left((2 r)^{2}-x^{2}\right)^{y-2} x^{2} d x=\frac{(2 r)^{2 y-1} \Gamma(1.5) \Gamma(y-1)}{2 \Gamma(y+0.5)}
$$

Substituting (6s) into (5s) and using $\Gamma(1.5)=0.5 \sqrt{ } \pi$ and $x \Gamma(x)=\Gamma(x+1)$, one obtains the target function for the average distance between nearest neighbors adsorbed on a sphere $(7 \mathrm{~s})$.

$$
\bar{x}=\sqrt{\pi}(y-1) r \frac{\Gamma(y-1)}{\Gamma(y+0.5)}=\sqrt{\pi} r \frac{\Gamma(y)}{\Gamma(y+0.5)}
$$

\section{Coverage as a function of particle size.}

The number of $\mathrm{Au}$ atoms in a particle with core radius $R$ is $\frac{4}{3} \pi R^{3} \bar{n}_{A u}^{f c c}$. Assuming Gaussian distribution of particle sizes, the number of Au particles with the radius between $R$ and $R+d R$ is $N_{p} \frac{e^{-(R-\bar{R})^{2} / 2 \sigma^{2}}}{\sigma \sqrt{2 \pi}} d R$. Here $N_{p}$ is the overall number of particles. The number of Au atoms in all particles with the radius between $R+d R$ is therefore $\frac{4}{3} \pi R^{3} \bar{n}_{A u}^{f c c} N_{p} \frac{e^{-(R-\bar{R})^{2} / 2 \sigma^{2}}}{\sigma \sqrt{2 \pi}} d R$, and the number of Au atoms in particles of all sizes is given by equation $(8 \mathrm{~s})$.

$$
N_{a t}=\int_{-\infty}^{\infty} \frac{4}{3} \pi R^{3} \bar{n}_{A u}^{f c c} \cdot N_{p} \cdot \frac{e^{-(R-\bar{R})^{2} / 2 \sigma^{2}}}{\sigma \sqrt{2 \pi}} d r=\frac{4 \pi \bar{n}_{A u}^{f c c} N_{p}}{3 \sigma \sqrt{2 \pi}} \cdot \int_{-\infty}^{\infty} R^{3} e^{-(R-\bar{R})^{2} / 2 \sigma^{2}} d R
$$

Let us define $z \equiv \frac{R-\bar{R}}{\sqrt{2} \sigma}$. The integral in equation (8s) can then be re-written as (9s)

$$
\int_{-\infty}^{\infty}(\sqrt{2} \sigma z+\bar{R})^{3} e^{-z^{2}} d z \sqrt{2} \sigma=4 \sigma^{4} \int_{-\infty}^{\infty} z^{3} e^{-z^{2}} d z+6 \sqrt{2} \sigma^{3} \bar{R} \int_{-\infty}^{\infty} z^{2} e^{-z^{2}} d z+6 \sigma^{2} \bar{R}^{2} \int_{-\infty}^{\infty} z e^{-z^{2}} d z+\sqrt{2} \sigma \bar{R}^{3} \int_{-\infty}^{\infty} e^{-z^{2}} d z
$$

The first and third integrals in function (9s) include odd functions. The integral of an odd function from $-\infty$ to $+\infty$ is zero. The second and fourth integrals are Gamma functions; the second integral is $\Gamma(1.5)=$ 
$\sqrt{ } \pi / 2$ and the fourth integral is $\Gamma(0.5)=\sqrt{ } \pi$. The integral (9s) is thus simplified to $3 \sqrt{2 \pi} \sigma^{3} \bar{R}+\sqrt{2 \pi} \sigma \bar{R}^{3}$. Substitution of this into equation (9s) gives equation (10s) which can be solved for $N_{p}$ to give (11s).

$$
\begin{aligned}
& N_{a t}=\frac{4}{3} \pi \bar{n}_{A u}^{f c c} N_{p}\left(3 \sigma^{2} \bar{R}+\bar{R}^{3}\right) \\
& N_{p}=\frac{3 N_{a t}}{4 \pi \bar{n}_{A u}^{f c c}\left(3 \sigma^{2} \bar{R}+\bar{R}^{3}\right)}
\end{aligned}
$$

The surface area of a particle with radius $R$ is $4 \pi R^{2}$. The surface area of all particles with the radius between $R+d R$ is therefore $4 \pi R^{2} N_{p} \frac{e^{-(R-\bar{R})^{2} / 2 \sigma^{2}}}{\sigma \sqrt{2 \pi}} d R$, and the surface area of all particles with any radius is given by equation (12s).

$$
A=\int_{-\infty}^{\infty} 4 \pi R^{2} \cdot N_{p} \cdot \frac{e^{-(R-\bar{R})^{2} / 2 \sigma^{2}}}{\sigma \sqrt{2 \pi}} d R=\frac{4 \pi N_{p}}{\sigma \sqrt{2 \pi}} \int_{-\infty}^{\infty} R^{2} e^{-(R-\bar{R})^{2} / 2 \sigma^{2}} d R
$$

Using our earlier definition of $z$, the integral in (12s) can be re-written as (13s)

$$
\int_{-\infty}^{\infty}(\sqrt{2} \sigma z+\bar{R})^{2} e^{-z^{2}} d z \sqrt{2} \sigma=2 \sqrt{2} \sigma^{3} \int_{-\infty}^{\infty} z^{2} e^{-z^{2}} d z+4 \sigma^{2} \bar{R} \int_{-\infty}^{\infty} z e^{-z^{2}} d z+\sqrt{2} \sigma \bar{R}^{2} \int_{-\infty}^{\infty} e^{-z^{2}} d z
$$

The second integral in function (13s) is zero (as it integrates an odd function). The first integral is $\Gamma(1.5)$ $=\sqrt{ } \pi / 2$ and the third integral is $\Gamma(0.5)=\sqrt{ } \pi$. The integral $(13 \mathrm{~s})$ is thus simplified to $\sqrt{2 \pi} \sigma^{3}+\sqrt{2 \pi} \sigma \bar{R}^{2}$. Substitution of this into equation (12s) gives equation (14s).

$$
A=4 \pi N_{p}\left(\sigma^{2}+\bar{R}^{2}\right)
$$

Substitution of equation (11s) into (14s) gives (15s).

$$
A=\frac{3 N_{a t}\left(\sigma^{2}+\bar{R}^{2}\right)}{\bar{n}_{A u}^{f c c}\left(3 \sigma^{2} \bar{R}+\bar{R}^{3}\right)}
$$

The average surface density of spin labels in nanoparticles is the ratio of the number of spin labels $N_{r}$ over the area $A$. For any nanoparticle with radius $R$, the average coverage $\bar{y}$ (e.g., the number of spin label per particle) equals the average surface density times the surface area of this nanoparticle (16s)

$$
\bar{y}=4 \pi R^{2} \frac{N_{r}}{A}=\frac{N_{r}}{N_{a t}} \cdot \frac{4 \pi R^{2} \bar{n}_{A u}^{f c c}\left(3 \sigma^{2} \bar{R}+\bar{R}^{3}\right)}{3\left(\sigma^{2}+\bar{R}^{2}\right)}
$$




\section{Number of Au atoms at the nanoparticle surface}

The total number of $\mathrm{Au}$ atoms in the particle is $\frac{4}{3} \pi R^{3} \bar{n}_{A u}^{f c c}$; assuming the hexagonal packing, the thickness of the outer "surface" layer of Au atoms is $2 R_{A u} \sqrt{ } 6 / 3$ (Figure $2 \mathrm{~s}$ ). The number of "core" atoms is thus $\frac{4}{3} \pi\left(R-\frac{2 \sqrt{6}}{3} R_{A u}\right)^{3} \bar{n}_{A u}^{f c c}$, and the number of "surface" atoms is given by equation $(17 \mathrm{~s})$.

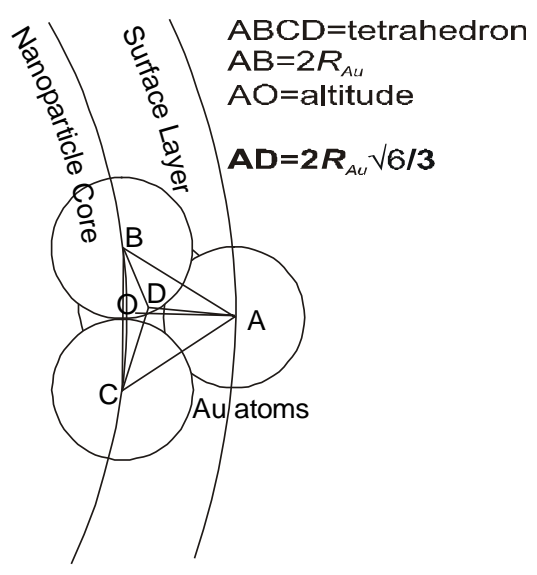

Figure 2s. FCC arrangement of the Au nanoparticle surface.

$$
n=\frac{4}{3} \pi \bar{n}_{A u}^{f c c}\left(R^{3}-\left(R-\frac{2 \sqrt{6}}{3} R_{A u}\right)^{3}\right)
$$

This simplistic approach assumes that the Au particles are spherical which is certainly not true. However formula (17s) predicts the number of surface Au atoms for regular icosahedra clusters ("magic number" clusters) remarkably well. In any case, it is only needed to estimate the maximum possible number of spin labels adsorbed on the same particle. The coverage of spin label used in this study was quite small (the maximum coverage was ca. 5\%); the probability of finding an Au particle with $n$ spin labels is therefore negligible, and a small error in determining the value of $n$ will have no impact on our calculations. We have checked that even substantial modification of equation $(17 \mathrm{~s})$ does not change the results of our calculations.

\section{Additivity of $d_{1} / d$ ratios.}

To further test the applicability of $d_{1} / d$ parameters to our system, we simulated powder EPR spectra for isolated nitroxides and spin-labeled $\mathrm{Au}$ nanoparticles.

The spectrum of isolated nitroxide radicals was simulated using EasySpin software developed by Dr Stefan Stoll (http://www.esr.ethz.ch/EasySpin/) with the following parameters:

g tensor principal values: 2.0088, 2.0061, 2.0027 
Line width: $0.75 \mathrm{mT}$

These values represent a typical nitroxide; we have made no attempts to fit this simulated spectrum to the experimental ones. The simulated spectrum is shown in Figure 4s (top spectrum).

To simulate the dipole-dipole interactions in spin-labelled Au nanoparticles, we employed an approach suggested by the H.-J. Steinhoff group (ref. 14 in the main manuscript). Briefly, the nitroxide alters the magnetic field on its neighbors by $\Delta \mathrm{B}$ as described by equation (18s)

$$
\Delta B= \pm \frac{3 g \beta}{4 x^{3}}\left(3 \cos ^{2} \theta-1\right)
$$

Here $g$ is the nitroxide $\mathrm{g}$-factor, $\beta$ is Bohr magneton, $\theta$ is the angle between the line connecting the interacting nitroxides and the direction of the external field, and $x$ is the distance between interacting nitroxides. Assuming random orientation of nitroxides in space, we used equation (18s) to calculate the spectral line shape ("Pake pattern") which was then convoluted with the simulated spectrum of isolated nitroxides. The angle $\theta$ was varied in the range $0-90^{\circ}$ with $0.75^{\circ}$ increments.

The results of this simulation for different interspin distances $x$ are shown in Figure $3 \mathrm{~s}$.

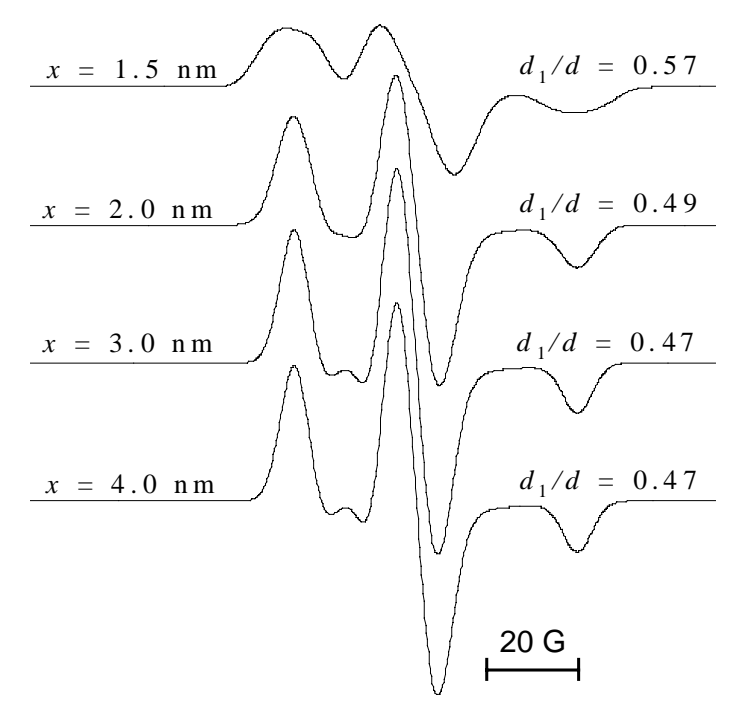

Figure 3s. Simulated EPR spectra for pairs of interacting nitroxides with different interspin distances.

One can see that the $d_{1} / d$ values decrease with increased interspin distance. However this effect becomes extremely small for interspin distances longer than $2 \mathrm{~nm}$. This proves that the $d_{1} / d$ values cannot be used for distances greater than $2 \mathrm{~nm}$.

We then used this simulation procedure to calculate the spectral line shape for Au nanoparticles labeled with 2, 3, 4 and 6 spin labels. The nanoparticle radius was assumed to be $1.45 \mathrm{~nm}$ (this is quite close to the experimental data, as the average radius of Au core is ca. $0.65 \mathrm{~nm}$ and the length of the ligand is ca. $0.8 \mathrm{~nm}$ ). The distribution of interspin distances was taken as described by equation (3) in the main manuscript (e.g., random distribution of spin labels on a sphere); the data were calculated with $0.05 \mathrm{~nm}$ interspin distance increment. The simulated data are shown in Figure 4s. 


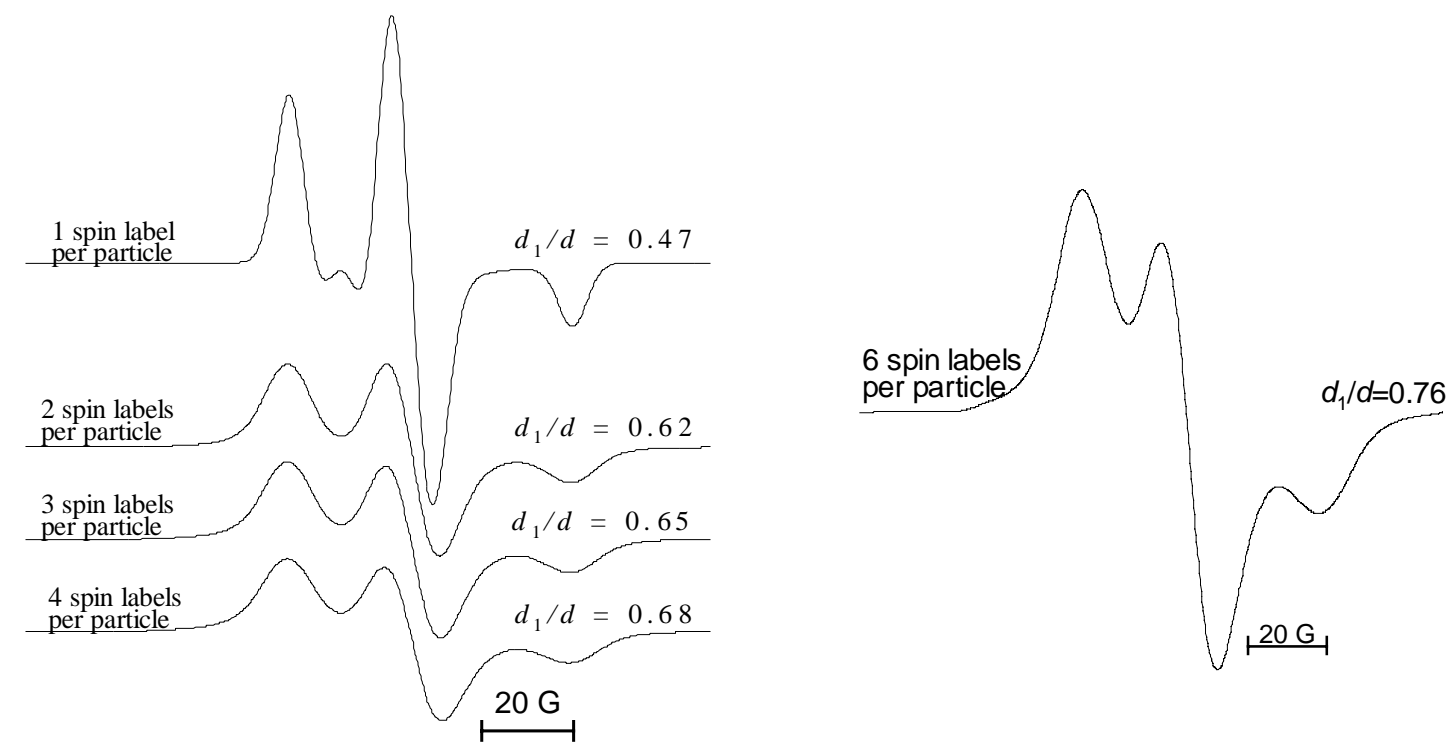

Figure 4s. Simulated EPR spectra of Au nanoparticles (monodisperse with $1.45 \mathrm{~nm}$ radius) possessing

\section{1, 2, 3, 4 and 6 spin labels per particle.}

To test the additivity of the $d_{1} / d$ values, we added pairs of spectra in Figure $4 \mathrm{~s}$ together in different proportions. The $d_{1} / d$ values for these sums of spectra were then compared with the values calculated using equation (13) in the main manuscript (Figure 5s). The value of parameter $\beta$ in equation (13) was taken as 0.307 (the same as in the main manuscript).

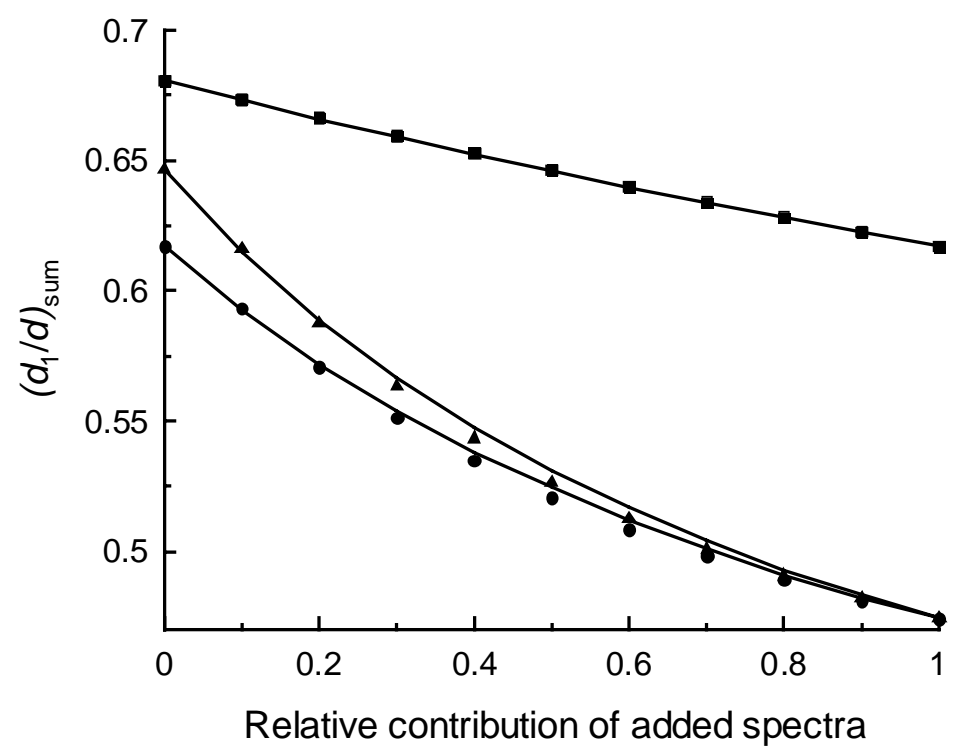

Figure 5s. $d_{1} / d$ Parameters for the sum of two simulated EPR spectra.

The excellent agreement between the calculated and simulated data confirms the additivity of the $d_{1} / d$ values and validates the use of equation (13) to calculate the $d_{1} / d$ values for sums of spectra. 